J. Lake Sci. (湖泊科学), 2017, 29(1): 1-8

DOI 10. 18307/2017. 0101

(C) 2017 by Journal of Lake Sciences

\title{
微囊藻毒素生物学功能研究进展”
}

\author{
甘南琴 ${ }^{1}$, 魏 念 $^{1,2}$, 宋立荣 ${ }^{* *}$ \\ (1: 中国科学院水生生物研究所,淡水生态与生物技术国家重点实验室, 武汉 430072) \\ (2: 中国科学院大学, 北京 100049)
}

\begin{abstract}
摘 要: 我国淡水水体蓝藻水华的频繁发生已成为我国目前和今后长时期内面临的重大水环境问题. 微囊藻 (Microcystis) 因其产生对人类健康具有危害的微囊藻毒素而尤其受到重视. 研究者对构成蓝藻水华的主要种类、微囊藻毒 素的毒性、毒理等方面的认识逐渐明晰, 但对微囊藻毒素生物学功能的了解还相对较少. 本文对微囊藻毒素的产生, 尤其 是近年来随着技术的发展在微囊藻毒素生物学功能方面的研究开展讨论: 总结了微囊藻毒素可能作为化感物质、参与光 合作用、在微囊藻的越冬或复苏中可能发挥的作用以及可能参与微囊藻群体形成及维持等 4 个方面的生物学功能; 分析 了微囊㩰毒素作为信号分子的研究现状; 探讨了全球气候变化及实验室模拟条件下微囊藻毒素产毒与无毒株的竞争, 并 对微囊藻毒素未来的研究热点及组学技术可能在其研究中的应用进行了展望.
\end{abstract}

关键词: 微囊藻毒素;生物学功能;微囊藻;蓝藻水华

\section{Recent progress in research of the biological function of microcystins}

\author{
GAN Nanqin ${ }^{1}$, WEI Nian ${ }^{1,2} \&$ SONG Lirong ${ }^{1 * *}$ \\ ( 1: State Key Laboratory of Freshwater Ecology and Biotechnology, Institute of Hydrobiology, Chinese Academy of Sciences, \\ Wuhan 430072, P.R. China) \\ (2: University of Chinese Academy of Sciences, Beijing 100049, P.R.China)
}

\begin{abstract}
Cyanobacterial bloom has become a frequent and problematic feature of many freshwater bodies over a wide geographical area, especially in China. Microcystis species are widely known for their production of the potent hepatotoxins called microcystins (MCs). While the toxicity of MCs on animals, including humans, has been well studied, the biological role of MCs on the organism from which they originate has not been clearly elucidated. In this paper, we summarized MCs production, especially MCs biological function with the development of technology in recent years. We reviewed and discussed the biological role of MCs in four aspects: Serving as allelochemical, involving in photosynthesis, taking part in the wintering and recovery of Microcystis and in the sustaining and formation of Microcystis colonies. In addition, MCs may act as signal molecule inside and outside Microcystis cells, relevant research has also been reviewed. Global climatic change which favour cyanobacterial blooms in eutrophic waters, may also influence the competition between MC-producing and non-MC-producing strains and their dominance. The study on the competition between MC-producing and non-MC-producing strains and the biological role of protein-bound MCs should be focused on for further investigation, fast development of variable -omics would favor the investigation.
\end{abstract}

Keywords: Microcystins; biological function; Microcystis; cyanobacterial bloom

近十多年来,我国众多淡水水体蓝藻水华的发生呈现出大范围、高频次、高生物量特征,引起水生态系 统结构和功能的失衡并直接或间接地影响到人类健康, 这已成为我国目前和今后长时期内面临的重大水环 境问题. 在世界范围内,蓝藻水华事件出现上升势头,其分布扩张范围扩大;在一些地区,每年蓝藻水华的暴 发和持续已成为常态. 微囊藻 (Microcystis) 是全球性广泛分布的水华蓝藻, 是我国水体中蓝藻水华的主要构

* 国家自然科学基金项目 (31370418,41561144008) 资助. 2016-05-13 收稿; $2016-10-09$ 收修改稿. 甘南琴 (1971 ), 女, 博士, 研究员; E-mail: gannq@ ihb.ac.cn.

** 通信作者;E-mail:lrsong@ihb.ac.cn. 
成种类, 因其产生对人类健康具有危害的蓝藻毒素——微囊藻毒素而尤其受到高度重视. 目前蓝藻毒素的 生态学功能是学术界关注的热点问题之一. 2013 年在南非召开的第九届产毒蓝藻国际大会上设立专题 “Natural function of cyanotoxin” 聚焦这一问题, 2016 年 10 月在武汉召开的第十届会议上,此问题仍然得到 关注.

我国湖泊发生的蓝藻水华中, 微囊藻是主要的水华优势种, 其产生的毒素为肝毒素微囊藻毒素 ( microcystins, MCs). 早期研究认为, 微囊藻毒素是一类次生代谢产物, 无明显的生理功能. 近年研究发现: 微囊藻 毒素可能参与胞内信号传递与基因调控 ${ }^{[1]}$; 高光强条件可促进微囊藻毒素与卡尔文循环等光合活性酶 (Calvin cycle enzymes) 的结合, 导致野生型比突变株 ( $\Delta m c y B$ mutant) 更加具有耐受高光强及氧化胁迫的优 势. 这些研究结果表明, 微囊藻毒素不是简单的次生代谢产物, 而是具有重要功能的细胞内化合物 ${ }^{[2]}$. 随着 微囊藻毒素与微囊藻细胞内多种蛋白质结合的发现, 微囊藻毒素生物学功能的研究进人了一个新的阶段. 本文在早期综述的基础上 ${ }^{[3]}$, 介绍微囊藻毒素的产生, 着重介绍微囊藻毒素的生物学功能及有毒、无毒株的 竞争,并简要评述该研究领域未来的发展趋势.

\section{1 微囊藻毒素的产生及影响因素}

研究发现并非所有的微囊藻藻株都能够产生微囊藻毒素, 微囊藻有毒株 (toxic strains) 与无毒株 (nontoxic strains) 的毒性由遗传决定. 为了证实这一观点, 遗传学家们开展了大量的微囊藻遗传结构研究, 有 关微囊藻毒素合成酶基因丛 ( $m c y$ clusters) 及其转录系统的研究也已日趋完善. Nishizawa 等 ${ }^{[4-5]}$ 首次发现了 3 个与微囊藻毒素合成有关的基因: $m c y A 、 m c y B$ 和 $m c y C$. 此后, 以篮选文库的方法克隆和测序了 $34 \mathrm{~kb}$ 的产 毒微囊藻 DNA 片断, 再次分离到 $m c y D 、 m c y E 、 m c y F$ 和 $m c y G 4$ 个基因. 这 4 个基因组成的操纵子位于 $m c y A$ 的上游, 与 $m c y A B C$ 组成的操纵子方向相反. Tillett 等 ${ }^{[6]}$ 通过基因突变和突变体分析, 在一 $55 \mathrm{~kb}$ 长的 DNA 片段中找到了 2 个操纵子, 由 10 个双向转录的开放阅读框组成, 即 $m c y A-C$ 和 $m c y D-J$.

微囊藻毒素的产生又受多种环境因素的影响, 如光照、温度、营养盐等, 其中光照是毒素产生的一个重 要制约因子. Utkilen 等 ${ }^{[7]}$ 研究发现, 在高光照条件下温度对毒素的产率几乎没有影响, 只有低光照条件下温 度才影响毒素的产率. 在一定的光照范围内, 微囊藻毒素的含量随着光强的增加而增加 ${ }^{[8]}$. 而在营养盐的研 究中发现,氮是微囊藻毒素含量最显著的调控因子 ${ }^{[9]}$. Harke 等 ${ }^{[10]}$ 对不同限制条件下细胞内转录组的变化 研究发现,仅在低氮条件下,所有微囊藻毒素合成基因 $(m c y A B C D E F)$ 均下调, 且单位细胞毒素含量与培养基 中无机氮浓度呈显著正相关. 另外, 氮调节蛋白 NtcA 可以结合到微囊藻毒素合成基因 $m c y A / D$ 的启动子区 域,参与微囊藻毒素的表达调控 ${ }^{[1-12]}$. 微量元素铁对微囊藻毒素含量也有显著影响, 降低铁的浓度会显著提 高毒素的产率 ${ }^{[13-14]}$. 与铁吸收相关的转录调节因子铁吸收调节蛋白 (ferric uptake regulator, Fur) 可以结合到 $m c y$ 基因的启动子区域 ${ }^{[15]}$, 如已经鉴定到的 FurA 可以结合到 $m c y S$ 的启动子区域 ${ }^{[16]}$, 表明 Fur 可能与缺铁 条件下毒素合成增加有关. Orr 等 ${ }^{[17]}$ 则提出微囊藻毒素的产生与微囊藻细胞的生长速率密切相关, 即微囊 藻毒素的产率在细胞指数生长初期达到最大, 在指数生长后期开始下降, 此结论随后得到多个实验室的 验证 ${ }^{[18-19]}$.

环境因素同时也影响 $m c y$ 基因的转录水平,其中研究较多的是光照及缺铁条件下 $m c y$ 基因转录水平的 调控. Sevilla 等 ${ }^{[13]}$ 研究发现, 铁饥饿状态能引起 $m c y$ 基因转录水平的显著提高和毒素含量的明显增加. Kaebernick 等 ${ }^{[20]}$ 的研究也发现, 高光强可引起 $m c y$ 基因转录水平显著提高, 但毒素含量无显著变化. Straub 等 ${ }^{[21]}$ 就此问题开展了深人研究,发现高光强条件下, $m c y$ 基因丛中 $m c y B$ 基因只上调了 $20 \%$, 而 $m c y A 、 m c y H$ 和 $m c y D$ 的转录水平分别上调了 $270 \% 、 330 \%$ 和 $370 \%$.

\section{2 微囊藻毒素的生物学功能}

目前, 对微囊藻毒素生物学功能的认识主要包括以下 4 个方面: (1) 微囊藻毒素作为化感物质抵御外界 侵扰; (2) 微囊藻毒素参与光合作用; (3) 微囊藻毒素可能对微囊藻的越冬或复苏起到一定作用; (4) 微囊藻 毒素可能参与微囊藻群体的形成及维持过程. 现分别归纳分析如下:

\section{1 微囊藻毒素作为化感物质抵御外界侵扰}

早期研究认为: 微囊藻毒素可能作为抵御外界侵扰的化感物质, 用于抑制其他光合自养生物的生长而 
利于自身的繁殖 ${ }^{[22]}$, 并且抵御浮游动物的摄食 ${ }^{[23]}$. Phelan 等 ${ }^{[24]}$ 研究发现外源添加的微囊藻毒素可以进人 集胞藻内并结合到内囊体膜上抑制集胞藻光合系统 II 的活性. Yang 等 ${ }^{[25]}$ 的实验结果表明, 铜绿微囊藻 $(M i-$ crocystis aeruginosa) 可能产生包括微囊藻毒素在内的化感物质抑制不产毒的惠氏微囊藻 (Microcystis wesenbergii) 的生长, 为野外水体蓝藻水华暴发期微囊藻毒素产毒株优势地位提供一定的解释. Schatz 等 ${ }^{[26]}$ 研究 则发现, 机械处理或者胁迫等条件下裂解细胞内释放的微囊藻毒素可被存活微囊藻细胞感知, 存活微囊藻 细胞进而显著提高微囊藻毒素基因表达量和微囊藻毒素含量适应胁迫环境, 表明微囊藻毒素可能作为种内 细胞交流的化感物质. 但是, 也有许多研究结果否定了微囊藻毒素作为化感物质的可能. 系统发育学研究发 现微囊藻毒素合成基因在蓝藻进化过程中一直存在, 微囊藻毒素合成早于水生动物的出现, 否定了微囊藻 毒素抵御浮游动物摄食的功能 ${ }^{[27]}$. Bártová 等 ${ }^{[28]}$ 研究发现在绿藻月牙藻 (Selenastrum bibraianum) 中添加纯 化的微囊藻毒素或铜绿微囊藻细胞粗提取液 (微囊藻毒素浓度为 $300 \mu \mathrm{g} / \mathrm{L}$ ) 均对月牙藻生长没有影响. 添加 微囊藻毒素甚至可能促进衣藻 (Chlamydomonas) 和小球藻 (Chlorella) 的生长 ${ }^{[29-30]} . \mathrm{Ma}^{\text {等 }}{ }^{[31]}$ 研究发现在水华 束丝藻 (Aphanizomenon flos-aquae) 培养物中添加微囊藻胞外提取物可以显著抑制水华束丝藻的生长但添加 纯化的 Microcystin-LR (MC-LR) 对水华束丝藻的生长并没有影响, 表明可能微囊藻毒素以外的代谢产物在 水华束丝藻生长抑制中发挥了作用. 微囊藻毒素作为化感物质的生物学功能有待商榷.

\section{2 微囊藻毒素参与光合作用}

在影响微囊藻毒素产生的环境因素中已提到, 光照是毒素产生的一个重要制约因子, 因此有科学家猜 测微囊藻毒素有可能在微囊藻的光合作用上具有一定的功能. 直到 2011 年, Dittmann 研究小组发现在高光 强下微囊藻毒素与卡尔文循环等光合活性酶相结合, 显示了野生型比突变株更加耐受高光强的优势 ${ }^{[32]}$. 同 时, 高光条件下微囊藻毒素与 RuBisCO 的结合更利于提高其氧化反应活性, 从而益于产毒株细胞降低细胞 内氧气浓度并更快耗费细胞内高光合速率积累的能量, 避免氧化损伤. 该研究直接证明了微囊藻毒素的胞 内功能, 对深人认识毒素的生物学功能做出了重要贡献. 代谢组学结果也表明, 在高光条件下, 微囊藻毒素 能协助微囊藻细胞更好地适应胁迫环境. 与无毒突变株相比, 高光条件下, 产毒株具有更高的光合效率, 也 更能耐受高光引起的培养液高 $\mathrm{pH}^{[33]}$.

另有学者认为, 微囊藻毒素在微囊藻指数生长期产量最高, 而在此时期, 营养盐相对限制, 光合作用可 利用的碳可能首先成为限制因子. 因此 Jähnichen 等 ${ }^{[34]}$ 猜测微囊藻毒素有可能参与 $\mathrm{CO}_{2}$ 浓缩机制 (Carbon Dioxide Concentrating Mechanism, CCM) , 从而在蓝藻细胞适应波动的无机碳时起到作用. 微囊藻毒素有毒株 在低 $\mathrm{CO}_{2}$ 条件下更具有竞争优势, 也可从侧面反映了微囊藻毒素可能在细胞适应低无机碳中发挥着重要作 用 ${ }^{[35]}$. 对 3 株有毒株和 3 株无毒株的蛋白组比较发现, 有毒株与无毒株的差异蛋白主要与碳一氮代谢和氧化 还原平衡有关, 这些差异蛋白就包括碳浓缩机制蛋白 $\mathrm{CcmK} 3$ 和 $\mathrm{CcmL}^{[36]}$. 这些结果均表明微囊藻毒素在碳 代谢和光合作用上具有重要的生物学功能.

\section{3 微囊藻毒素可能对微囊藻的越冬或复苏起到一定作用}

Ihle 等 ${ }^{[37]}$ 对微囊藻生活史的 4 个阶段(复苏 (reinvasion)、生长 (pelagic growth)、沉降( sedimentation)、越 冬 (overwintering) ) 中底泥里微囊藻毒素及微囊藻生物量连续 3 年监测发现: 复苏阶段毒素及藻生物量均减 少, 沉降阶段毒素及藻生物量增加, 但整个生长或越冬时期, 毒素含量及藻生物量保持相对稳定状态. 由此 推断, 微囊藻毒素在细胞越冬时未释放, 可能对微囊藻的越冬或复苏起了某种作用. Schatz 等 ${ }^{[26]}$ 研究也发现 正在分解的微囊藻细胞释放的微囊藻毒素可增强细胞的聚集, 改进其他存活细胞的适应性. 但 Misson 等 ${ }^{[38]}$ 在研究毒素对微囊藻复苏的影响时并未发现高产毒微囊藻藻株具有较高的复苏率, 相反毒素含量越低的藻 株复苏率越高, 并且添加毒素会降低微囊藻的复苏率. 因此, 有关微囊藻毒素在微囊藻的越冬或复苏中起到 一定作用的说法还存在争议,有待进一步研究.

\section{4 微囊藻毒素可能参与微囊藻群体的形成及维持过程}

Jungmann 等 ${ }^{\left[{ }^{[39}\right]}$ 在研究自然水体环境因子对微囊藻毒素合成的影响中, 发现常规的多个理化因子与蓝 藻毒素浓度无显著相关性; 但根据体积将微囊藻群体分为不同组分, 发现群体越大, 毒素含量越高, 这是有 关自然水华微囊藻群体与毒素相关性的首次报道. Kurmayer 等 ${ }^{[40]}$ 对野外单个群体微囊藻的尺寸大小及产 毒量分析发现: 产毒微囊藻的产毒量与群体大小呈正相关. 这些发现与微囊藻群体形成中群体胶被的解释 
是相吻合的. Kehr 等 ${ }^{[1]}$ 则试图从分子水平加以探讨: 外加过量表达的 MVN ( microvirin, 一种凝集素) 于 MVN 突变株中观察到明显的细胞聚集 ( cell aggregates), 这一现象说明微囊藻细胞中含有的 MVN 可能参与了微 囊藻细胞间的识别及微囊藻群体的形成. 与此同时, MVN 与微囊藻毒素关系研究中发现, MVN 缺失突变株 中微囊藻毒素含量明显减少, 而微囊藻毒素缺失突变株中 MVN 的表达异常, 表明微囊藻毒素与 MVN 之间 很可能存在功能上的相互关系. Sedmak 等 ${ }^{[41]}$ 发现, 在培养条件下, 微囊藻毒素可诱导微囊藻及栅藻 (Scenedesmus) 等浮游植物形态及生理的改变, 促进单细胞微囊藻聚集为小群体. 我们实验室前期研究发现: 环境剂量微囊藻毒素 (环境中通常可检测到的微囊藻毒素浓度 $(0.25 \sim 1 \mu \mathrm{g} / \mathrm{L}$ )) 可显著增大微囊藻群体的尺 寸(约 3 倍以上), 而及时、持续地清除释放到胞外的微囊藻毒素分子, 微囊藻群体的尺寸则会显著减小, 表 明天然环境浓度下的微囊藻毒素对微囊藻群体形态的维持具有重要作用 ${ }^{[42]}$. 在野外环境中, 鞭毛虫的摄食 和高光强可能是微囊藻群体形成最主要的原因, 其他因素如营养盐限制 ${ }^{[43]}$ 、附生和浮游细菌 ${ }^{[44]}$ 可能也参与 群体的形成和维持. 在野外水体中尤其在水华期间, 环境剂量的微囊藻毒素可能更多地参与微囊藻群体的 维持,从而保持其在水华期间的竞争优势.

\section{3 微囊藻毒素作为信号分子的研究现状}

关于微囊藻毒素作为信号分子的说法主要基于以下 3 篇报道: (1) 德国 Dittmann 研究小组的发现: 微囊 藻毒素可能参与胞内信号传递与基因调控 ${ }^{[1]}$; (2) 以色列研究小组发现正在分解的微囊藻细胞释放的微囊 藻毒素或可作为微囊藻群落的信息化学物质 (infochemicals), 增强细胞的聚集, 改进其他存活细胞的适应 性 $^{[21]}$; (3) 我们近期研究发现, 产毒微囊藻细胞在生长过程中释放到胞外的微囊藻毒素具有信号物质的功 能, 微囊藻毒素可通过激活产毒及非产毒微囊藻细胞中部分与多糖合成相关基因的表达, 诱导一系列胞外 多糖产物的释放,进而促进微囊藻群体的聚集 ${ }^{[42]}$.

在近期对 PCC7806 及其 $m c y B$ 无毒突变株的转录组研究中发现, 突变株 pksI-pksIII 基因簇表达量显著高 于有毒株. 在突变株中外源添加终浓度为 $50 \mathrm{ng} / \mathrm{ml}$ 的 MC-LR 后, 突变株中 pksI-pksIII 基因簇表达量下调, 且 该基因簇对外源 MC-LR 的响应是高度特异性的. 表明外源的 MC-LR 可以调控细胞内的信号通路, 此结果也 进一步证实了微囊藻毒素的信号分子作用 ${ }^{[45]}$.

Jüttner 等 ${ }^{[46]}$ 发现微囊藻毒素可与藻胆素 (Phycobilins) 结合, 有利于提高其在细胞内的浓度, 且与蛋白 质结合的微囊藻毒素在纯化时无法与 C18 萃取小柱结合, 可能导致微囊藻毒素的定量偏差. 使用抗微囊藻 毒素抗体, Vela 等 ${ }^{[47]}$ 进一步发现微囊藻毒素可与藻细胞内的多种蛋白质结合. 还原剂 DTT 和表面离子活性 剂 SDS 均对结合无影响, 微囊澡毒素的结合不改变结合蛋白质的等电性质. 比较产微囊藻毒素与产微囊藻 毒素缺失株,Zilliges 等 ${ }^{[32}$ 发现在产微囊藻毒素细胞内, 微囊藻毒素可以与特定蛋白质结合, 并证明微囊藻 毒素与特定蛋白质之间的结合方式和微囊澡毒素与 PP1 的结合方式一样, 即通过 Mdha 残基与特定蛋白质 Cys 残基形成硫梄键而共价结合.

Meissner 等 ${ }^{[48]}$ 进一步研究发现藻细胞中约有 30\% 40\%的微囊藻毒素与蛋白质结合. 在高光条件下, 与 特定蛋白质结合的微囊藻毒素增多至 $50 \% \sim 60 \%$, 其占细胞总微囊藻毒素的比例甚至可能超过游离的微囊 藻毒素. 而在此条件下, 细胞中游离的微囊藻毒素含量并未发生显著性改变, 进一步表明与蛋白质结合的微 囊藻毒素可能在细胞中具有重要作用.

\section{4 微囊藻有毒与无毒株的竞争}

目前有关微囊藻有毒与无毒株的竞争主要集中在实验室内改变培养条件下的研究: 如, 改变培养温度, 发现温度升高 $\left(+4^{\circ} \mathrm{C}\right)$ 可以促进产毒株在水华群体中占更大的比例, 或者促进细胞合成更多毒素合成 $m c y D$ 基因. 升高的温度结合富营养化 (磷浓度升高) 促进产毒结果更显著, 推测随着全球温室效应和富营养化, 水 体水华趋于具有更高毒性 ${ }^{[49]}$. 伴随着臭氧层的破坏, 到达地面的紫外辐射也日益增强, Ding 等 ${ }^{[50]}$ 研究发现, 无毒株对 UVB 辐射更敏感, 受 UVB 辐射后其恢复率也更低; 相对无毒株, 产毒株更能耐受环境剂量的 UVB 辐射, 微囊藻毒素可能协助有毒株抵御 UVB 辐射造成的氧化胁迫, 环境 UVB 辐射的增加可能显著改变蓝藻 水华组成和多样性而有利于产毒株在水华期间的主导地位. Van de Waal 等 ${ }^{[35]}$ 则发现有毒微囊藻株与无毒 
株在无机碳的利用方面存在差异, 有毒株较无毒株在适应低浓度 $\mathrm{CO}_{2}$ 条件时更具优势. 由此推断, 随着环境 $\mathrm{CO}_{2}$ 浓度的升高, 有害藻类水华的群落组成及毒性有可能发生改变. 李伟等 ${ }^{[51]}$ 人工模拟酸雨研究了不同 $\mathrm{pH}$ 处理后产毒株和无毒株的光合生理变化及其对紫外辐射的敏感性, 发现产毒株较无毒株更能耐受酸雨和紫 外辐射. 其结果也表明随着未来全球环境的变化, 产毒株可能更具有优势. Wang 等 ${ }^{[52]}$ 研究结果则显示光合 作用在产毒株与无毒株的演替中起到了重要的作用. 作者选取了 3 株有毒株和 3 株无毒株, 发现在不同营 养水平和高光条件下, 产毒株与无毒株的光合系统响应存在明显的差异, 产毒株和无毒株在水华不同时期 光合效率的不同导致其优势差异. Lei 等 ${ }^{[53]}$ 研究了不同温度、光照及不同氮磷浓度条件产毒株与无毒株的 竞争, 发现虽然单培养条件下无毒株生长速率高于产毒株, 但在同样条件的产毒株与无毒株共培养条件下 产毒株占据明显的优势.

Briand 等 ${ }^{[54]}$ 的研究结果表明在营养限制的条件如氮限制、低温低光照条件下产毒株占据明显的优势, 但是在营养充足的条件下在与有毒株的混合培养中则是无毒株占据优势, 表明环境条件差异可能导致产毒 与无毒株的优势差异. 使用产毒株及其 $m c y B$ 突变株的进一步实验结果 ${ }^{[55]}$ 表明产毒株在营养充足条件下失 去优势地位可能与其在营养充足条件下产生毒素所耗费的能量有关. 在营养充足的条件下, 产生毒素动用 相关细胞内代谢过程及耗费的能量可能大于毒素在产毒株中具有的相关生物学功能优势, 从而导致无毒株 在混合培养中占据优势. 在另外一个采用不同产毒株与无毒株混培来比较不同光强条件下产毒株与无毒株 竞争优势的研究 ${ }^{[56]}$ 中, 产毒株在不同光强条件下均占据一定的优势, 这可能与实验所选取的株系差异有关.

我们实验室研究了近 3 年太湖北部湖区优势藻类演替及其理化参数的动力学, 并比对历史数据, 提出 近 10 年来太湖优势微囊藻种类的演替格局基本一致, 水华暴发期产毒藻类的比例在 45\% 55\% 之间波动, 单位细胞毒素浓度保持相对稳定 ${ }^{[57]}$.

\section{5 展望}

\section{1 全球环境变化下微囊藻有毒与无毒株的竞争演替格局}

分析近 10 年来发表的太湖蓝藻水华优势种群演替和蓝藻毒素浓度的监测数据, 得出如下结论: 近 10 年 来, 太湖北部区域营养盐浓度尤其是总氮浓度有所下降. 但太湖北部区域优势水华的产毒能力仍维持较高 水平, 蓝藻毒素浓度未明显下降, 局部区域存在较高的风险. 随着全球气候的变化, 微囊藻有毒与无毒株的 竞争演替格局是否会发生变化将成为关注的焦点,这一格局的变化又会对人类和生态环境起到怎样的影响 也将是备受关注的环境问题.

\section{2 微囊藻毒素与细胞内蛋白质结合}

微囊藻毒素与 Calvin Cycle 重要蛋白质 RuBisCO 的结合直接证明了微囊藻毒素在细胞内所发挥的作 用 ${ }^{[32]}$. 我们实验前期研究发现不同生长条件下微囊藻毒素结合的蛋白质存在差异, 这种差异可能与微囊藻 的生存策略有关. 蛋白质是细胞代谢通路的直接功能行使者, 微囊藻毒素与蛋白质结合可以稳定其化学构 象从而维持其功能, 这些蛋白质的功能直接反映了微囊藻毒素所具有的生物学功能, 微囊藻毒素结合蛋白 质的研究也将是未来的重要研究方向.

\section{3 组学技术的应用}

已有的转录组学、代谢组学和蛋白组学结果提示微囊藻毒素有毒株相比无毒株在 $\mathrm{C} / \mathrm{N}$ 代谢途径上具有 一定的差异, 在耐受高光强上具有明显的优势, 为微囊藻毒素生物学功能研究提供了丰富的数据和理论依 据. 各种组学的结合应用将有利于对细胞内各种表达水平 (DNA、RNA、蛋白质和代谢物) 上的变化与信号的 传递形成更加整体和精确的认识, 转录组学、蛋白组学、翻译后修饰组学及代谢组学等技术在相关研究中的 应用将在微囊藻毒素生物学功能研究中起到重要作用. 宏基因组学和宏蛋白组学等技术则将对了解微囊藻 毒素在有毒与无毒株之间竞争所起到的作用及对产毒株在野外水体中所占据的优势提供丰富的信息.

\section{6 参考文献}

[ 1 ] Kehr J, Zilliges Y, Springer A et al. A mannan binding lectin is involved in cell-cell attachment in a toxic strain of Microcystis aeruginosa. Mol Microbiol, 2006, 59(3): 893-906. 
[ 2 ] Kaplan A, Harel M, Kaplan-Levy RN et al. The languages spoken in the water body (or the biological role of cyanobacterial toxins). Front Microbiol, 2012, 3: 138.

[ 3 ] Song Lirong, Chen Wei. Production of microcystins in bloom-forming cyanobacteria and their environmental fates: A review. J Lake Sci, 2009, 21(6): 749-757. DOI: 10.18307/2009.0601. [ 宋立荣, 陈伟. 水华蓝藻产毒的生物学机制及 毒素的环境归趋研究进展. 湖泊科学, 2009,21(6) : 749-757.]

[ 4 ] Nishizawa T, Asayama M, Fujii K et al. Genetic analysis of the peptide synthetase genes for a cyclic heptapeptide microcysin in Microcystis spp. J Biochem, 1999, 126(3) : 520-529.

[ 5 ] Nishizawa T, Ueda A, Asayama M et al. Polyketide synthase gene coupled to the peptide synthetase module involved in the biosynthesis of the cyclic heptapeptide microcystin. J Biochem, 2000, 127(5) : 779-789.

[ 6 ] Tillett D, Dittmann E, Erhard M et al. Structural organization of microcystin biosynthesis in Microcystis aeruginosa PCC7806, an integrated peptide-polyketide synthetase system. Chem Biol, 2000, 7(10) : 753-764.

[ 7 ] Utkilen H, Gjølme N. Toxin production by Microcystis aeruginosa as a function of light in continuous cultures and its ecological significance. Appl Environ Microbiol, 1992, 58(4) : 13-21.

[ 8 ] Wiedner C, Visser PM, Fastner J et al. Effects of light on the microcystin content of Microcystis strain PCC7806. Appl Environ Microbiol, 2003, 69(3): 1475-1481.

[ 9 ] Downing TG, Meyer C, Gehringer MM et al. Microcystin content of Microcystis aeruginosa is modulated by nitrogen uptake rate relative to specific growth rate or carbon fixation rate. Environ Toxicol, 2005, 20(3) : 257-262.

[10] Harke MJ, Gobler CJ. Global transcriptional responses of the toxic cyanobacterium, Microcystis aeruginosa, to nitrogen stress, phosphorus stress, and the growth on organic matter. PLoS One, 2013, 8(7): e69834.

[11] Ginn HP, Pearson LA, Neilan BA. NtcA from Microcystis aeruginosa PCC7806 is autoregulatory and binds to the microcystin promoter. Appl Environ Microbiol, 2010, 76(13) : 4362-4368.

[12] Kuniyoshi TM, Gonzalez A, Lopez-Gomollon S et al. 2-oxoglutarate enhances NtcA binding activity to promoter regions of the microcystin synthesis gene cluster. FEBS Lett, 2011, 585(24) : 3921-3926.

[13 ] Sevilla E, Martin-Luna B, Vela L et al. Iron availability affects $m c y D$ expression and microcystin-LR synthesis in Microcystis aeruginosa PCC7806. Environ Microbiol, 2008, 10(10) : 2476-2483.

[14] Alexova R, Fujii M, Birch D et al. Iron uptake and toxin synthesis in the bloom-forming Microcystis aeruginosa under iron limitation. Environ Microbiol, 2011, 13(4) : 1064-1077.

[15] Martin-Luna B, Sevilla E, Hernandez JA et al. Fur from Microcystis aeruginosa binds in vitro promoter regions of the microcystin biosynthesis gene cluster. Phytochem, 2006, 67(9) : 876-881.

[16] Martin-Luna B, Hernandez JA, Bes MT et al. Identification of a ferric uptake regulator from Microcystis aeruginosa PCC7806. FEMS Microbiol Lett, 2006, 254(1): 63-70.

[17] Orr P, Jones G. Relationship between microcystin production and cell division rates in nitrogen-limited Microcystis aeruginosa cultures. Limnol Oceanogr, 1998, 43(7):1604-1614.

[18] Long BM, Jones GJ, Orr PT. Cellular microcystin content in N-limited Microcystis aeruginosa can be predicted from growth rate. Appl Environ Microbiol, 2001, 67(1) : 278-283.

[19] Vezie C, Rapala J, Vaitomaa J et al. Effect of nitrogen and phosphorus on growth of toxic and nontoxic Microcystis strains and on intracellular microcystin concentrations. Microb Ecol, 2002, 43(4) : 443-454.

[20] Kaebernick M, Dittmann E, Böerner T et al. Multiple alternate transcripts direct the biosynthesis of microcystin, a cyanobacterial nonribosomal peptide. Appl Environ Microbiol, 2002, 68(2) : 449-455.

[21] Straub C, Quillardet P, Vergalli J et al. A day in the life of Microcystis aeruginosa PCC7806 as revealed by a transcriptomic analysis. PLoS One, 2011, 6(1): e16208.

[22] Pflugmacher S. Possible allelopathic effects of cyanotoxin, with reference to microcystin-LR, in aquatic ecosystems. Environ Toxicol, 2002, 17(4): 407-413.

[23] Lampert W. Inhibitory and toxic effects of blue-green algae on Daphnia. Int Rev Ges Hydro boil, 1981, 66( 3) : $285-298$.

[24] Phelan RR, Downing TG. The localization of exogenous microcystin LR taken up by a non-microcystin producing cyanobacterium. Toxicon, 2014, 89: 87-90.

[25] Yang J, Deng X, Xian Q et al. Allelopathic effect of Microcystis aeruginosa on Microcystis wesenbergii: Microcystin-LR as a potential allelochemical. Hydrobiologia, 2014, 727(1) : 65-73. 
[26] Schatz D, Keren Y, Vardi A et al. Towards clarification of the biological role of microcystins, a family of cyanobacterial toxins. Environ Microbiol, 2007, 9(4) : 965-970.

[27] Rantala A, Fewer DP, Hisbergues M et al. Phylogenetic evidence for the early evolution of microcystin synthesis. Proc Natl Acad Sci USA, 2004, 101(2): 568-573.

[28] Bártová K, Hilscherová K, Babica $\mathrm{P}$ et al. Effects of microcystin and complex cyanobacterial samples on the growth and oxidative stress parameters in green alga Pseudokirchneriella subcapitata and comparison with the model oxidative stressorherbicide paraquat. Environ Toxicol, 2010, 26(6) : 641-648.

[29] Pinheiro C, Azevedo J, Campos A et al. Absence of negative allelopathic effects of cylindrospermopsin and microcystin-LR on selected marine and freshwater phytoplankton species. Hydrobiologia, 2013, 705(1) : 27-42.

[30] Pinheiro C, Azevedo J, Campos A et al. The interactive effects of microcystin-LR and cylindrospermopsin on the growth rate of the freshwater algae Chlorella vulgaris. Ecotoxicology, 2016, 25(4) : 745-758.

[31] Ma H, Wu Y, Gan N et al. Growth inhibitory effect of Microcystis on Aphanizomenon flos-aquae isolated from cyanobacteria bloom in Lake Dianchi, China. Harmful Algae, 2015, 42: 43-51.

[32] Zilliges Y, Kehr JC, Meissner S et al. The cyanobacterial hepatotoxin microcystin binds to proteins and increases the fitness of Microcystis under oxidative stress conditions. PLoS One, 2011, 6(3) : e17615.

[33] Meissner S, Steinhauser D, Dittmann E. Metabolomic analysis indicates a pivotal role of the hepatotoxin microcystin in high light adaptation of Microcystis. Environ Microbial, 2014, 17(5) : 1497-1509.

[34] Jähnichen S, Ihle T, Petzoldt T et al. Impact of inorganic carbon availability on microcystin production by Microcystis aeruginosa PCC7806. Appl Environ Microbiol, 2007, 73(21) : 6994-7002.

[ 35 ] Van de Waal D, Verspagen J, Finke J et al. Reversal in competitive dominance of a toxic versus non-toxic cyanobacterium in response to rising $\mathrm{CO}_{2}$. ISME J, 2011, 5(9): 1438-1450.

[36] Alexova R, Haynes PA, Ferrari BC et al. Comparative protein expression in different strains of the bloom-forming cyanobacterium Microcystis aeruginosa. Mol Cell Proteomics, 2011, 10(9): M110.003749.

[37] Ihle T, Sabine J, Jurgen B. Wax and Wane of Microcystis (Cyanophyceae) and microcystins in lake sediments: A case study in Quitzdorf reservoir (Germany). J Phycol, 2005, 41(3) : 479-488.

[38 ] Misson B, Sabart M, Amblard C et al. Involvement of microcystins and colony size in the benthic recruitment of the cyanobacterium Microcystis (Cyanophyceae). J Phycol, 2011, 47(1) : 42-51.

[39] Jungmann D, Ludwichowski KU, Faltin V et al. A field study to investigate environmental factors that could effect microcystin synthesis of a Microcystis population in the Bautzen reservoir. Int Revue Ges Hydrobiol, 1996, 81(4) : 493-501.

[ 40] Kurmayer R, Christiansen G, Chorus I. The abundance of microcystin-producing genotypes correlates positively with colony size in Microcystis sp. and determines its microcystin net production in Lake Wannsee. Appl Environ Microbiol, 2003, 69 (2) : 787-795.

[41] Sedmak B, Eleršek T. Microcystins induce morphological and physiological changes in selected representative phytoplanktons. Microb Ecol, 2006, 51(4) : 508-515.

[ 42] Gan N, Xiao Y, Zhu L et al. The role of microcystins in maintaining colonies of bloom-forming Microcystis spp. Environ Microbiol, 2012, 14(3): 730-742.

[43] Ma J, Brookes JD, Qin B et al. Environmental factors controlling colony formation in blooms of the cyanobacteria Microcystis spp. in Lake Taihu, China. Harmful Algae, 2014, 31: 136-142.

[44] Shen H, Niu Y, Xie P et al. Morphological and physiological changes in Microcystis aeruginosa as a result of interactions with heterotrophic bacteria. Freshw Biol, 2011, 56(6): 1065-1080.

[45] Makower AK, Schuurmans JM, Groth D et al. Transcriptomics-aided dissection of the intracellular and extracellular roles of microcystin in Microcystis aeruginosa PCC 7806. Appl Environ Microbiol, 2015, 81(2) : 544-554.

[46] Jüttner F, Lüthi H. Topology and enhanced toxicity of bound microcystins in Microcystis PCC 7806. Toxicon, 2008,51 (3) : 388-397.

[47] Vela L, Sevilla E, Gonzalez C et al. Exploring the interaction of microcystin-LR with proteins and DNA. Toxicol in Vitro, $2008,22(7): 1714-1718$.

[48] Meissner S, Fastner J, Dittmann E. Microcystin production revisited: Conjugate formation makes a major contribution. Environ Microbiol, 2013, 15(6) : 1810-1820. 
[49] Davis TW, Berry DL, Boyer GL et al. The effects of temperature and nutrients on the growth and dynamics of toxic and non-toxic strains of Microcystis during cyanobacteria blooms. Harmful Algae, 2009, 8: 715-725.

[50] Ding Y, Song L, Sedmak B. UVB radiation as a potential selective factor favoring microcystin producing bloom forming cyanobacteria. PLoS One, 2013, 8: e73919.

[51] Li Wei, Yang Yuling, Huang Song et al. Physiological responses of toxigenic and non-toxigenic strains of Microcystis aeruginosa to simulated acid rain and UV radiation. Acta Ecologica Sinica, 2015, 35(23): 7615-7624. [李伟, 杨雨玲, 黄松 等. 产毒与不产毒铜绿微囊藻对模拟酸雨及紫外辐射的生理响应. 生态学报, 2015, 35(23): 7615-7624.]

[52] Wang Z, Li D, Cao X et al. Photosynthesis regulates succession of toxic and nontoxic strains in blooms of Microcystis (Cyanobacteria). Phycologia, 2015, 54(6): 640-648.

[53] Lei L, Li C, Peng L et al. Competition between toxic and non-toxic Microcystis aeruginosa and its ecological implication. Ecotoxicology, 2015, 24(7/8) : 1411-1418.

[54] Briand E, Yéprémian C, Humbert JF et al. Competition between microcystin- and non-microcystin-producing Planktothrix agardhii (cyanobacteria) strains under different environmental conditions. Environ Microbiol, 2008, 10( 12) : 3337-3348.

[55] Briand E, Bormans M, Quiblier C et al. Evidence of the cost of the production of microcystins by Microcystis aeruginosa under differing light and nitrate environmental conditions. PLoS One, 2012, 7(1) : e29981.

[56] Renaud SL, Pick FR, Fortin N. Effect of light intensity on the relative dominance of toxigenic and nontoxigenic strains of Microcystis aeruginosa. Appl Environ Microbiol, 2011, 77(19) : 7016-7022.

[57] Hu L, Shan K, Lin L et al. Multi-year assessment of toxic genotypes and microcystin concentration in northern Lake Taihu, China. Toxins, 2016, 8(1): 23. 